\title{
Entropic uncertainty measures for large dimensional hydrogenic systems
}

D. Puertas-Centeno, N. M. Temme, I. V. Toranzo, and J. S. Dehesa

Citation: Journal of Mathematical Physics 58, 103302 (2017);

View online: https://doi.org/10.1063/1.5006569

View Table of Contents: http://aip.scitation.org/toc/jmp/58/10

Published by the American Institute of Physics

\section{Articles you may be interested in}

Rigged configuration descriptions of the crystals $B(\infty)$ and $B(\lambda)$ for special linear Lie algebras Journal of Mathematical Physics 58, 101701 (2017); 10.1063/1.4986276

A new algorithm for computing branching rules and Clebsch-Gordan coefficients of unitary representations of compact groups

Journal of Mathematical Physics 58, 101702 (2017); 10.1063/1.5004259

Response to "Comment on 'Entropy and complexity analysis of hydrogenic Rydberg atoms"' [J. Math. Phys. 58, 104101 (2017)]

Journal of Mathematical Physics 58, 104102 (2017); 10.1063/1.4986518

Comment on "Entropy and complexity analysis of hydrogenic Rydberg atoms" [J. Math. Phys. 54, 052109 (2013)]

Journal of Mathematical Physics 58, 104101 (2017); 10.1063/1.5006191

Abelian Turaev-Virelizier theorem and U(1) BF surgery formulas

Journal of Mathematical Physics 58, 102301 (2017); 10.1063/1.4986850

Aharonov-Bohm effect without contact with the solenoid

Journal of Mathematical Physics 58, 102102 (2017); 10.1063/1.4992123

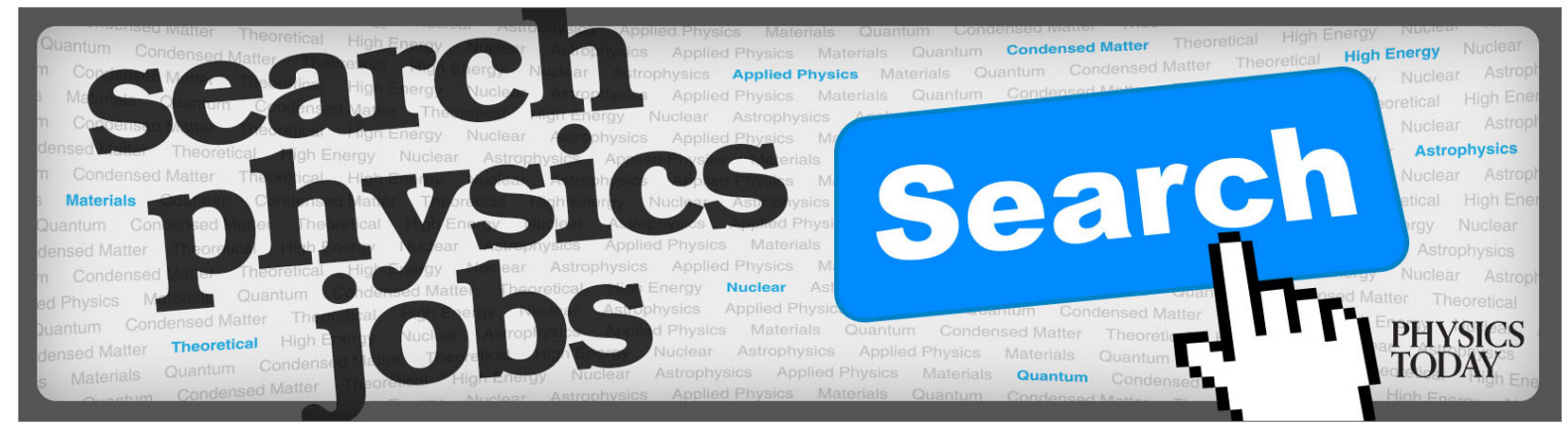




\title{
Entropic uncertainty measures for large dimensional hydrogenic systems
}

\author{
D. Puertas-Centeno, ${ }^{1, a)}$ N. M. Temme, ${ }^{2, b)}$ I. V. Toranzo, ${ }^{1, c)}$ \\ and J. S. Dehesa ${ }^{1, d)}$ \\ ${ }^{1}$ Departamento de Física Atómica, Molecular y Nuclear, Universidad de Granada, \\ Granada 18071, Spain and Instituto Carlos I de Física Teórica y Computacional, \\ Universidad de Granada, Granada 18071, Spain \\ ${ }^{2}$ IAA, 1825 BD 25 Alkmaar, The Netherlands
}

(Received 12 January 2017; accepted 25 September 2017; published online 11 October 2017)

\begin{abstract}
The entropic moments of the probability density of a quantum system in position and momentum spaces describe not only some fundamental and/or experimentally accessible quantities of the system but also the entropic uncertainty measures of Rényi type, which allow one to find the most relevant mathematical formalizations of the position-momentum Heisenberg's uncertainty principle, the entropic uncertainty relations. It is known that the solution of difficult three-dimensional problems can be very well approximated by a series development in $1 / D$ in similar systems with a nonstandard dimensionality $D$; moreover, several physical quantities of numerous atomic and molecular systems have been numerically shown to have values in the large$D$ limit comparable to the corresponding ones provided by the three-dimensional numerical self-consistent field methods. The $D$-dimensional hydrogenic atom is the main prototype of the physics of multidimensional many-electron systems. In this work, we rigorously determine the leading term of the Rényi entropies of the $D$ dimensional hydrogenic atom at the limit of large $D$. As a byproduct, we show that our results saturate the known position-momentum Rényi-entropy-based uncertainty relations. Published by AIP Publishing. https://doi.org/10.1063/1.5006569
\end{abstract}

\section{INTRODUCTION}

In an excellent tutorial article about quarks, gluons, and impossible problems of quantum chromodynamics, Edward Witten ${ }^{1}$ illustrated the utility of the large-dimension $D$ limit with a rough calculation for helium. This prompted Dudley R. Herschbach et al. ${ }^{3,4}$ and other authors (see the review $^{2}$ ) to develop a new strategy, the dimensional scaling method, to solve first the quantum problems with one degree of freedom ${ }^{2}$ and later the much more difficult Coulomb problems involving two or more non-separable, strongly coupled degrees of freedom which usually take place in physics of atoms and molecules. ${ }^{3,4}$ With this method, a finite many-body problem is typically solved in the large- $D$ limit, most often in an analytical way, and then perturbation theory in $1 / D$ is used to obtain an approximate result for the standard dimension $(D=3)$.

Physics in the large- $D$ limit becomes much simpler. Indeed, in this limit, the electrons of a many-electron system assume fixed positions relative to the nuclei and each other, in the $D$-scaled space. Moreover, the large- $D$ electronic geometry and energy correspond to the minimum of an exactly known effective potential and can be determined from classical electrostatics for any atom or molecule, what remembers the prequantum models of Lewis and Langmuir. ${ }^{5,6}$ The $(D \rightarrow \infty)$-limit is called pseudoclassical, tantamount to $h \rightarrow 0$ and/or $m_{e} \rightarrow \infty$ in the kinetic energy. This limit is not

\footnotetext{
a)vidda@correo.ugr.es

b) Former address: CWI, Science Park 123, 1098 XG Amsterdam, The Netherlands. Electronic mail: Nico.Temme@ cwi.nl.

c)ivtoranzo@ugr.es

d) dehesa@ugr.es
} 
the same as the conventional classical limit obtained by $h \rightarrow 0$ for a fixed dimension. ${ }^{7,8}$ Although at first sight the electrons at rest in fixed locations might seem violate the uncertainty principle, this is not true because that occurs only in the $D$-scaled space (see, e.g., Ref. 6). For $D$, finite but very large, the electrons are confined to harmonic oscillations about the fixed positions attained in the $(D \rightarrow \infty)$-limit. Moreover, the large- $D$ limit of numerous physical properties of almost all atoms with up to 100 electrons and many diatomic molecules have been numerically evaluated, obtaining values comparable to or better than single-zeta Hartree-Fock calculations. ${ }^{3,4,9}$

The main prototype of the $D$-dimensional Coulomb many-body systems, the $D$-dimensional hydrogen atom (i.e., a negatively charged particle moving in a space of $D$ dimensions around a positively charged core which electromagnetically binds it in its orbit), has been investigated in detail starting from its wave functions which are analytically known ${ }^{10-12}$ in the two conjugated position and momentum spaces for any dimension. This system includes a wide variety of physical objects, such as hydrogenic atoms and ions, some exotic atoms and antimatter atoms, excitons in semiconductors and qubits.

The spreading properties of the electronic distribution of the $D$-dimensional hydrogenic atom have been analyzed by means of its moments around the origin (radial expectation values) in both position ${ }^{13-18}$ and momentum ${ }^{19,20}$ spaces. However, these quantities are formally given in terms of $D$, the hyperquantum numbers of the hydrogenic states and the nuclear charge $Z$ through a generalized hypergeometric function ${ }_{p+1} F_{p}(1)$, which cannot be easily calculated unless the hyperquantum numbers and/or the dimension $D$ are sufficiently small. Recently the position and momentum moments around the origin of the $D$-dimensional hydrogenic atom have been determined in a simple and compact form for the highly and very highly excited (i.e., Rydberg) states ${ }^{12,21}$ as well as for any excited state at large $D . .^{22}$

The determination of the entropic measures of the $D$-dimensional hydrogenic atom, which describe most appropriately the electronic uncertainty of the system, is far more difficult except for the lowest-lying energy states despite some efforts. ${ }^{12}$ This is because these quantities are described by means of some power or logarithmic functionals of the electron density, which cannot be calculated in an analytical way nor numerically computed; the latter is basically because a naive numerical evaluation using quadratures is not convenient due to the increasing number of integrable singularities when the principal hyperquantum number $n$ is increasing, which spoils any attempt to achieve reasonable accuracy even for rather small $n \cdot{ }^{23}$ Recently, the main entropic properties of the $D$-dimensional Rydberg hydrogenic states (namely, the Rényi, Shannon, and Tsallis entropies) have been explicitly calculated in a compact form ${ }^{24,25}$ by use of modern techniques of approximation theory based on the strong asymptotics $(n \rightarrow \infty)$ of the Laguerre $\mathcal{L}_{n}^{(\alpha)}(x)$ and Gegenbauer $\mathcal{C}_{n}^{(\alpha)}(x)$ polynomials which control the state's wave functions in position and momentum spaces, respectively. ${ }^{26}$

In this work, we first determine the Rényi entropy and then we conjecture the Shannon entropy in both position and momentum spaces for the large-dimensional hydrogenic states in terms of the dimensionality $D$, the nuclear charge $Z$ and the principal and orbital hyperquantum numbers of the states. The Rényi entropies $R_{q}[\rho], q>0$ are defined ${ }^{27,28}$ as

$$
R_{q}[\rho]=\frac{1}{1-q} \log \int_{\mathbb{R}^{D}}[\rho(\vec{r})]^{q} d \vec{r}, \quad q \neq 1 .
$$

Note that the Shannon entropy $S[\rho]=-\int \rho(\vec{r}) \log \rho(\vec{r}) d \vec{r}=\lim _{q \rightarrow 1} R_{q}[\rho]$; see, e.g., Ref. 29. These quantities completely characterize the density $\rho(\vec{r})^{30}$ under certain conditions. In fact, we can calculate from (1) other relevant entropic quantities such as the disequilibrium $\langle\rho\rangle=\exp \left(-R_{2}[\rho]\right)$, and the Tsallis entropies $T_{q}[\rho]=\frac{1}{q-1}\left(1-\int_{\mathbb{R}^{D}}[\rho(\vec{r})]^{q} d \vec{r}\right), q>0^{31}$ as

$$
T_{q}[\rho]=\frac{1}{1-q}\left[e^{(1-q) R_{q}[\rho]}-1\right],
$$

which holds for $q \neq 1$. Here again, the Shannon entropy $S[\rho]=\lim _{q \rightarrow 1} T_{q}[\rho]$. The properties of the Rényi entropies and their applications have been widely considered; see, e.g., Refs. 29, 32, and 33 and the reviews. ${ }^{34-36}$ The use of Rényi and Shannon entropies as measures of uncertainty allow a wider quantitative range of applicability than the moments around the origin and the standard 
or root-square-mean deviation do. This permits, for example, a quantitative discussion of quantum uncertainty relations further beyond the conventional Heisenberg-like uncertainty relations. ${ }^{22,34,35,37}$

The structure of this work is the following. In Sec. II, the wave functions of the $D$-dimensional hydrogenic states in both position and momentum spaces are briefly described, and the corresponding probability densities are given. In Sec. III, we determine the physical Rényi entropies of the $D$-dimensional hydrogenic atom at large $D$ by use of some recent theorems relative to the asymptotics $(\alpha \rightarrow \infty)$ of the underlying Rényi-like integral functionals of Laguerre polynomials $\mathcal{L}_{k}^{(\alpha)}(x)$ and Gegenbauer polynomials $\mathcal{C}_{k}^{(\alpha)}(x)$, which control the hydrogenic wavefunctions as described in Sec. II. The dominant term of the joint position-momentum uncertainty sum for the general states of the large dimensional hydrogenic systems is also given and, what is most interesting, shown to saturate the known position-momentum Rényi-entropy-based uncertainty relations. ${ }^{38-40}$ Finally, some conclusions, open problems, and three appendixes are given.

\section{THE D-DIMENSIONAL HYDROGENIC PROBLEM: BASICS}

In this section, we briefly summarize the physical solutions of the Schrödinger equation of the $D$ dimensional hydrogenic system in both position and momentum spaces. Then we give the associated position and momentum $D$-dimensional probability densities of the system.

The time-independent Schrödinger equation of a $D$-dimensional $(D>1)$ hydrogenic system (i.e., an electron moving under the action of the $D$-dimensional Coulomb potential $\left.V(\vec{r})=-\frac{Z}{r}\right)$ is given by

$$
\left(-\frac{1}{2} \vec{\nabla}_{D}^{2}-\frac{Z}{r}\right) \Psi(\vec{r})=E \Psi(\vec{r})
$$

where $\vec{\nabla}_{D}$ denotes the $D$-dimensional gradient operator, $Z$ is the nuclear charge, and the electronic position vector $\vec{r}=\left(x_{1}, \ldots, x_{D}\right)$ in hyperspherical units is given as $\left(r, \theta_{1}, \theta_{2}, \ldots, \theta_{D-1}\right) \equiv\left(r, \Omega_{D-1}\right)$, $\Omega_{D-1} \in S^{D-1}$, where $r \equiv|\vec{r}|=\sqrt{\sum_{i=1}^{D} x_{i}^{2}} \in[0,+\infty)$ and $x_{i}=r\left(\prod_{k=1}^{i-1} \sin \theta_{k}\right) \cos \theta_{i}$ for $1 \leq i \leq D$ and with $\theta_{i} \in[0, \pi), i<D-1, \theta_{D-1} \equiv \phi \in[0,2 \pi)$. It is assumed that the nucleus is located at the origin and, by convention, $\theta_{D}=0$ and the empty product is the unity. Atomic units are used throughout the paper

It is known ${ }^{10-12}$ that the energies belonging to the discrete spectrum are given by

$$
E=-\frac{Z^{2}}{2 \eta^{2}}, \eta=n+\frac{D-3}{2} ; n=1,2,3, \ldots,
$$

and the associated eigenfunction can be expressed as

$$
\Psi_{n, l,\{\mu\}}(\vec{r})=\mathcal{R}_{n, l}(r) \mathcal{Y}_{l,\{\mu\}}\left(\Omega_{D-1}\right),
$$

where $(l,\{\mu\}) \equiv\left(l \equiv \mu_{1}, \mu_{2}, \ldots, \mu_{D-1}\right)$ denote the hyperquantum numbers associated with the angular variables $\Omega_{D-1} \equiv\left(\theta_{1}, \theta_{2}, \ldots, \theta_{D-1}\right)$, which may take all values consistent with the inequalities $l \equiv \mu_{1}$ $\geq \mu_{2} \geq \cdots \geq\left|\mu_{D-1}\right| \equiv|m| \geq 0$. The radial eigenfunction is given by

$$
\begin{aligned}
\mathcal{R}_{n, l}(r) & =\mathfrak{N}_{n, l}\left(\frac{r}{\lambda}\right)^{l} e^{-\frac{r}{2 \lambda}} \mathcal{L}_{n-l-1}^{(2 l+D-2)}\left(\frac{r}{\lambda}\right) \\
& =\mathfrak{N}_{n, l}\left[\frac{\omega_{2 L+1}(\tilde{r})}{\tilde{r}^{D-2}}\right]^{1 / 2} \mathcal{L}_{\eta-L-1}^{(2 L+1)}(\tilde{r}) \\
& =\left(\frac{\lambda^{-D}}{2 \eta}\right)^{1 / 2}\left[\frac{\omega_{2 L+1}(\tilde{r})}{\tilde{r}^{D-2}}\right]^{1 / 2} \widehat{\mathcal{L}}_{\eta-L-1}^{(2 L+1)}(\tilde{r}),
\end{aligned}
$$

where the "grand orbital angular momentum quantum number" $L$ and the dimensionless parameter $\tilde{r}$ are

$$
\begin{aligned}
& L=l+\frac{D-3}{2}, \quad l=0,1,2, \ldots, \\
& \tilde{r}=\frac{r}{\lambda}, \quad \lambda=\frac{\eta}{2 Z},
\end{aligned}
$$


and $\omega_{\alpha}(x)=x^{\alpha} e^{-x}, \alpha=2 L+1=2 l+D-2$, is the weight function of the Laguerre polynomials $\mathcal{L}_{k}^{(\alpha)}(x), x \in[0, \infty)$. Note that $\alpha \geq 0$ for $D \geq 2$. The symbols $\mathcal{L}_{k}^{\left(\alpha^{\prime}\right)}(x)$ and $\widehat{\mathcal{L}}_{k}^{\left(\alpha^{\prime}\right)}(x)$ denote the orthogonal and orthonormal Laguerre polynomials, respectively, so that

$$
\widehat{\mathcal{L}}_{k}^{\left(\alpha^{\prime}\right)}(x)=\left(\frac{k !}{\Gamma\left(k+\alpha^{\prime}+1\right)}\right)^{1 / 2} \mathcal{L}_{k}^{\left(\alpha^{\prime}\right)}(x)
$$

for any parameter $\alpha^{\prime}>-1$, and finally

$$
\mathfrak{N}_{n, l} \equiv \lambda^{-\frac{D}{2}}\left\{\frac{(\eta-L-1) !}{2 \eta(\eta+L) !}\right\}^{\frac{1}{2}}=\left\{\left(\frac{2 Z}{n+\frac{D-3}{2}}\right)^{D} \frac{(n-l-1) !}{2\left(n+\frac{D-3}{2}\right)(n+l+D-3) !}\right\}^{\frac{1}{2}}
$$

represents the normalization constant which ensures that $\int\left|\Psi_{\eta, l,\{\mu\}}(\vec{r})\right|^{2} d \vec{r}=1$. Note that the $D$ dimensional volume element is $d \vec{r} \equiv d^{D} r=r^{D-1} d r d \Omega_{D-1}$ and

$$
d \Omega_{D-1}=\left(\prod_{j=1}^{D-2}\left(\sin \theta_{j}\right)^{2 \alpha_{j}} d \theta_{j}\right) d \theta_{D-1}
$$

where $2 \alpha_{j}=D-j-1$. The angular eigenfunctions are the hyperspherical harmonics, $\mathcal{Y}_{l,\{\mu\}}\left(\Omega_{D-1}\right)$, defined $^{10,12,42}$ as

$$
\mathcal{Y}_{l,\{\mu\}}\left(\Omega_{D-1}\right)=\mathcal{N}_{l,\{\mu\}} e^{i m \phi} \times \prod_{j=1}^{D-2} \mathcal{C}_{\mu_{j}-\mu_{j+1}}^{\left(\alpha_{j}+\mu_{j+1}\right)}\left(\cos \theta_{j}\right)\left(\sin \theta_{j}\right)^{\mu_{j+1}}
$$

with the squared normalization constant given as

$$
\begin{aligned}
\mathcal{N}_{l,\{\mu\}}^{2} & =\frac{1}{2 \pi} \prod_{j=1}^{D-2} \frac{\left(\alpha_{j}+\mu_{j}\right)\left(\mu_{j}-\mu_{j+1}\right) !\left[\Gamma\left(\alpha_{j}+\mu_{j+1}\right)\right]^{2}}{\pi 2^{1-2 \alpha_{j}-2 \mu_{j+1}} \Gamma\left(2 \alpha_{j}+\mu_{j}+\mu_{j+1}\right)}, \\
& =\frac{1}{2 \pi} \prod_{j=1}^{D-2} A_{\mu_{j}, \mu_{j+1}}^{(j)},
\end{aligned}
$$

where the symbol $\mathcal{C}_{k}^{\left(\alpha^{\prime}\right)}(t)$ denotes the Gegenbauer polynomial ${ }^{41}$ of degree $k$ and parameter $\alpha^{\prime}$.

Then, the quantum probability density of a $D$-dimensional hydrogenic stationary state $(n, l,\{\mu\})$ is given in position space by the squared modulus of the position eigenfunction given by (5) as

$$
\rho_{n, l,\{\mu\}}(\vec{r})=\rho_{n, l}(\tilde{r})\left|\mathcal{Y}_{l,\{\mu\}}\left(\Omega_{D-1}\right)\right|^{2},
$$

where the radial part of the density is the univariate radial density function,

$$
\rho_{n, l}(\tilde{r})=\left[\mathcal{R}_{n, l}(r)\right]^{2}=\frac{\lambda^{-D}}{2 \eta} \frac{\omega_{2 L+1}(\tilde{r})}{\tilde{r}^{D-2}}\left[\widehat{\mathcal{L}}_{\eta-L-1}^{(2 L+1)}(\tilde{r})\right]^{2} .
$$

On the other hand, the Fourier transform of the position eigenfunction $\Psi_{\eta, l,\{\mu\}}(\vec{r})$ given by (5) provides the eigenfunction of the system in the conjugated momentum space as

$$
\tilde{\Psi}_{n, l,\{\mu\}}(\vec{p})=\mathcal{M}_{n, l}(p) \mathcal{Y}_{l,\{\mu\}}\left(\Omega_{D-1}\right),
$$

where the radial part is

$$
\mathcal{M}_{n, l}(p)=K_{n, l} \frac{(\eta \tilde{p})^{l}}{\left(1+\eta^{2} \tilde{p}^{2}\right)^{L+2}} \mathcal{C}_{\eta-L-1}^{(L+1)}\left(\frac{1-\eta^{2} \tilde{p}^{2}}{1+\eta^{2} \tilde{p}^{2}}\right)
$$

with $\tilde{p}=\frac{p}{Z}$ and the normalization constant

$$
K_{n, l}=Z^{-\frac{D}{2}} 2^{2 L+3}\left[\frac{(\eta-L-1) !}{2 \pi(\eta+L) !}\right]^{\frac{1}{2}} \Gamma(L+1) \eta^{\frac{D+1}{2}} .
$$


Then, the expression

$$
\begin{aligned}
\gamma_{n, l,\{\mu\}}(\vec{p}) & =\left|\tilde{\Psi}_{n, l,\{\mu\}}(\vec{p})\right|^{2}=\mathcal{M}_{n, l}^{2}(p)\left|\mathcal{Y}_{l,\{\mu\}}\left(\Omega_{D-1}\right)\right|^{2} \\
& =K_{n, l}^{2} \frac{(\eta \tilde{p})^{2 l}}{\left(1+\eta^{2} \tilde{p}^{2}\right)^{2 L+4}}\left[\mathcal{C}_{\eta-L-1}^{(L+1)}\left(\frac{1-\eta^{2} \tilde{p}^{2}}{1+\eta^{2} \tilde{p}^{2}}\right)\right]^{2}\left|\mathcal{Y}_{l,\{\mu\}}\left(\Omega_{D-1}\right)\right|^{2}
\end{aligned}
$$

gives the momentum probability density of the $D$-dimensional hydrogenic stationary state with the hyperquantum numbers $(n, l,\{\mu\})$.

\section{RÉNYI ENTROPIES OF LARGE DIMENSIONAL HYDROGENIC STATES}

In this section, we obtain the Rényi entropies of a generic $D$-dimensional hydrogenic state $(n, l$, $\{\mu\})$ in the large- $D$ limit in both position and momentum spaces. We start with the expressions (13) and (18) of the position and momentum probability densities of the system, respectively.

To calculate the position Rényi entropy, we decompose it into radial and angular parts. The radial part is first expressed in terms of a Rényi-like integral functional of Laguerre polynomials $\mathcal{L}_{n-l-1}^{(\alpha)}(x)$ with $\alpha=D+2 l-2$, and then this functional is determined in the large- $D$ limit by means of Theorem 1 (see Appendix A). The angular part is given by a Rényi-like integral functional of hyperspherical harmonics, which can be expressed in terms of Rényi-like functionals of Gegenbauer polynomials $\mathcal{C}_{n-l-1}^{\left(\alpha^{\prime \prime}\right)}$ with $\alpha^{\prime \prime}=D / 2+l-1 / 2$; later on, we evaluate these Gegenbauer functionals at large $D$ by means of Theorem 2 (see Appendix B), with emphasis in the circular and $(n s)$ states which are characterized by the hyperquantum numbers $(n, l=n-1,\{\mu\}=\{n-1\})$ and $(n, l=0,\{\mu\}=\{0\})$, respectively.

Operating similarly in momentum space, we can determine the momentum Rényi entropy of the system. In this space, both the radial and angular parts of the momentum wave functions of the hydrogenic states are controlled by Gegenbauer polynomials as follows from Sec. II. Consequently, the two radial and angular contributions to the momentum Rényi entropy are expressed in terms of Rényi-like functionals of Gegenbauer polynomials.

\section{A. Rényi entropy in position space}

Let us obtain the position Rényi entropy of the probability density $\rho_{n, l,\{\mu\}}(\vec{r})$ given by (13), which according to (1) is defined as

$$
R_{q}\left[\rho_{n, l,\{\mu\}}\right]=\frac{1}{1-q} \log W_{q}\left[\rho_{n, l,\{\mu\}}\right] ; \quad 0<q<\infty, q \neq 1,
$$

where the symbol $W_{q}\left[\rho_{n, l,\{\mu\}}\right]$ denotes the entropic moments of the density

$$
\begin{aligned}
W_{q}\left[\rho_{n, l,\{\mu\}}\right] & =\int_{\mathbb{R}^{D}}\left[\rho_{n, l,\{\mu\}}(\vec{r})\right]^{q} d \vec{r} \\
& =\int_{0}^{\infty}\left[\rho_{n, l}(\tilde{r})\right]^{q} r^{D-1} d r \times \Lambda_{l,\{\mu\}}\left(\Omega_{D-1}\right),
\end{aligned}
$$

with the angular part given by

$$
\Lambda_{l,\{\mu\}}\left(\Omega_{D-1}\right)=\int_{S^{D-1}}\left|\mathcal{Y}_{l,\{\mu\}}\left(\Omega_{D-1}\right)\right|^{2 q} d \Omega_{D-1} .
$$

Then, from Eqs. (20) and (19), we can obtain the Rényi entropies of the $D$-dimensional hydrogenic state $(n, l,\{\mu\})$ as follows:

$$
R_{q}\left[\rho_{n, l,\{\mu\}}\right]=R_{q}\left[\rho_{n, l}\right]+R_{q}\left[\mathcal{Y}_{l,\{\mu\}}\right],
$$

where $R_{q}\left[\rho_{n, l}\right]$ denotes the radial part,

$$
R_{q}\left[\rho_{n, l}\right]=\frac{1}{1-q} \log \int_{0}^{\infty}\left[\rho_{n, l}(\tilde{r})\right]^{q} r^{D-1} d r,
$$

and $R_{q}\left[\mathcal{Y}_{l,\{\mu\}}\right]$ denotes the angular part,

$$
R_{q}\left[\mathcal{Y}_{l,\{\mu\}}\right]=\frac{1}{1-q} \log \Lambda_{l,\{\mu\}}\left(\Omega_{D-1}\right) .
$$


Here our aim is to determine the large- $D$ behavior of the Rényi entropy $R_{q}\left[\rho_{n, l,\{\mu\}}\right]$ when all the hyperquantum numbers are fixed. According to (22), this issue requires the knowledge at $D>>1$ of the radial and angular Rényi entropies, i.e., $R_{q}\left[\rho_{n, l}\right]$ and $R_{q}\left[\mathcal{Y}_{l,\{\mu\}}\right]$, respectively, whose determination is done in Secs. III A 1 and III A 2.

\section{Radial position Rényi entropy}

According to Eq. (23), the radial Rényi entropy can be expressed as

$$
R_{q}\left[\rho_{n, l}\right]=\frac{1}{1-q} \log \left[\frac{\eta^{D(1-q)-q}}{2^{D(1-q)+q} Z^{D(1-q)}} N_{n, l}(D, q)\right],
$$

where $N_{n, l}(D, q)$ denotes the following $\mathfrak{Q}_{q}$-norm of the Laguerre polynomials,

$$
N_{n, l}(D, q)=\int_{0}^{\infty}\left(\left[\widehat{\mathcal{L}}_{n-l-1}^{(\alpha)}(x)\right]^{2} w_{\alpha}(x)\right)^{q} x^{\beta} d x
$$

with $\tilde{r} \equiv x$ and

$$
\alpha=D+2 l-2, l=0,1,2, \ldots, n-1, q>0 \text { and } \beta=(2-D) q+D-1 .
$$

We note that (27) guarantees the convergence of integral (26); i.e., the condition $\beta+q \alpha=2 l q+D-1$ $>-1$ is always satisfied for physically meaningful values of the parameters. Moreover, the norm $N_{n, l}(D, q)$ can be rewritten as

$$
\begin{aligned}
N_{n, l}(D, q) & =\int_{0}^{\infty}\left(\left[\widehat{\mathcal{L}}_{n-l-1}^{(D+2 l-2)}(x)\right]^{2} w_{D+2 l-2}(x)\right)^{q} x^{2 q-1+(1-q) D} d x \\
& =\left[\frac{\Gamma(n-l)}{\Gamma(n+l+D-2)}\right]^{q} \int_{0}^{\infty} x^{D+2 l q-1} e^{-q x}\left[\mathcal{L}_{n-l-1}^{(D+2 l-2)}(x)\right]^{2 q} d x .
\end{aligned}
$$

Then, the determination of the large- $D$ behavior of the radial Rényi entropy $R_{q}\left[\rho_{n, l}\right]$ requires the calculation of the asymptotics of the Laguerre functional $N_{n, l}(D, q)$ defined by (26); that is, the evaluation of the Rényi-like integral functional given by (28) when $D>>1$. We do it by applying Theorem 1 (see Appendix A) to the functional $N_{n, l}(D, q)$ given by $(28)$ with $(n, l)$ fixed, obtaining for every non-negative $q \neq 1$ that

$$
\begin{aligned}
N_{n, l}(D, q) \sim & {\left[\frac{\Gamma(n-l)}{\Gamma(n+l+D-2)}\right]^{q} \frac{\sqrt{2 \pi}}{[\Gamma(n-l)]^{2 q}}|q-1|^{2(n-l-1) q} } \\
& \times e^{-D-2 l+2}(D+2(l-1))^{D+2 q(n-1)-\frac{1}{2}} q^{-D-2 q(n-1)} \\
= & \frac{\sqrt{2 \pi}|q-1|^{2(n-l-1) q}}{\Gamma(n-l)^{q}} e^{-D-2 l+2} \frac{(D+2 l-2)^{D+2 q(n-1)-\frac{1}{2}} q^{-D-2 q(n-1)}}{\Gamma(D+n+l-2)^{q}} \\
\sim & \frac{(2 \pi)^{\frac{1-q}{2}}|q-1|^{2(n-l-1) q}}{\Gamma(n-l)^{q}} q^{-2 q(n-1)}\left(\frac{D}{e}\right)^{D(1-q)} q^{-D} D^{q\left(n-l+\frac{1}{2}\right)-\frac{1}{2}},
\end{aligned}
$$

where we have used the Stirling's formula ${ }^{41}$ for the gamma function $\Gamma(x)=e^{-x} x^{x-\frac{1}{2}}(2 \pi)^{\frac{1}{2}}$ $\left[1+\mathcal{O}\left(x^{-1}\right)\right]$.

Then, Eqs. (25)-(29) allow us to find the following large- $D$ behavior for the radial Rényi entropy:

$$
\begin{aligned}
R_{q}\left[\rho_{n, l}\right] & \sim \frac{1}{1-q} \log \left\{\frac{(2 \pi)^{\frac{1-q}{2}}|q-1|^{2(n-l-1) q}}{\Gamma(n-l)^{q}} q^{-2 q(n-1)} \frac{D^{D(1-q)-q} e^{(2 n-3)(1-q)}}{4^{D(1-q)} Z^{D(1-q)}}\left(\frac{D}{e}\right)^{D(1-q)} q^{-D} D^{q\left(n-l+\frac{1}{2}\right)-\frac{1}{2}}\right\} \\
& =\frac{1}{1-q} \log \left\{\frac{(2 \pi)^{\frac{1-q}{2}}|q-1|^{2(n-l-1) q}}{\Gamma(n-l)^{q}} \frac{e^{(2 n-3)(1-q)}}{q^{2 q(n-1)}}\left(\frac{D^{2}}{4 Z e}\right)^{D(1-q)} q^{-D} D^{q\left(n-l-\frac{1}{2}\right)-\frac{1}{2}}\right\},
\end{aligned}
$$

which can be rewritten as

$$
R_{q}\left[\rho_{n, l}\right] \sim 2 D \log [D]+D \log \left[\frac{q^{\frac{1}{q-1}}}{4 Z e}\right]+\frac{q\left(n-l-\frac{1}{2}\right)-\frac{1}{2}}{1-q} \log D+\frac{1}{1-q} \log \mathcal{F}(n, l, q),
$$


where $\mathcal{F}(n, l, q)=\frac{(2 \pi)^{\frac{1-q}{2}}|q-1|^{2(n-l-1) q}}{\Gamma(n-l)^{q}} \frac{e^{(2 n-3)(1-q)}}{q^{2 q(n-1)}}$. Further terms in this asymptotic expansion can be obtained by means of Theorem 1 (see Appendix A).

Note that, since $q^{\frac{1}{q-1}} \rightarrow e$ when $q \rightarrow 1$, we have the following conjecture for the value of the Shannon entropy:

$$
S\left[\rho_{n, l}\right] \sim 2 D \log [D]-D \log [4 Z],
$$

which can be numerically shown to be correct. However a more rigorous expression for this quantity remains to be proved.

Then, according to Eq. (22), to fix the total Rényi entropy $R_{q}\left[\rho_{n, l,\{\mu\}}\right]$, at large $D$, it only remains the evaluation of the corresponding large- $D$ behavior of the angular part $R_{q}\left[\mathcal{Y}_{l,\{\mu\}}\right]$, which will be done in Sec. III A 2.

\section{Angular Rényi entropy}

Here we will effort to calculate the large- $D$ behavior of the angular part $R_{q}\left[\mathcal{Y}_{l,\{\mu\}}\right]$ of the total position and momentum Rényi entropies defined by Eq. (24). Therein, according to (11) and (21), the Rényi-like functional $\Lambda_{l,\{\mu\}}\left(\Omega_{D-1}\right)$ of the hypersherical harmonics can be expressed as

$$
\begin{aligned}
\Lambda_{l,\{\mu\}}\left(\Omega_{D-1}\right) & =\int_{S^{D-1}}\left|\mathcal{Y}_{l,\{\mu\}}\left(\Omega_{D-1}\right)\right|^{2 q} d \Omega_{D-1} \\
& =\mathcal{N}_{l,\{\mu\}}^{2 q} \int_{S^{D-1}} \prod_{j=1}^{D-2}\left[C_{\mu_{j}-\mu_{j+1}}^{\left(\alpha_{j}+\mu_{j+1}\right)}\left(\cos \theta_{j}\right)\right]^{2 q}\left(\sin \theta_{j}\right)^{2 q \mu_{j+1}} d \Omega_{D-1} \\
& =2 \pi \mathcal{N}_{l,\{\mu\}}^{2 q} \prod_{j=1}^{D-2} \int_{0}^{\pi}\left[C_{\mu_{j}-\mu_{j+1}}^{\left(\alpha_{j}+\mu_{j+1}\right)}\left(\cos \theta_{j}\right)\right]^{2 q}\left(\sin \theta_{j}\right)^{2 q \mu_{j+1}+2 \alpha_{j}} d \theta_{j},
\end{aligned}
$$

where the normalization constant $\mathcal{N}_{l,\{\mu\}}$ is given by (12). Moreover, note that the integrals within the product are Rényi-like functionals of Gegenbauer polynomials of the type considered in Theorem 2 (see Appendix B).

To calculate the dominant term of $\Lambda_{l,\{\mu\}}\left(\Omega_{D-1}\right)$ at large $D$, we use Theorem 2 at zeroth order or, what is equivalent, we use the following limiting expressions of the Gegenbauer polynomials to monomials [see Ref. 41, Eq. (18.6.4)]:

$$
\lim _{\alpha^{\prime} \rightarrow \infty}\left(2 \alpha^{\prime}\right)^{-k} \mathcal{C}_{k}^{\left(\alpha^{\prime}\right)}(x)=\frac{x^{k}}{k !}
$$

which allows us to find

$$
\begin{aligned}
\Lambda_{l,\{\mu\}}\left(\Omega_{D-1}\right) \sim & 2 \pi \mathcal{N}_{l,\{\mu\}}^{2 q} \prod_{j=1}^{D-2} \frac{\left[2\left(\alpha_{j}+\mu_{j+1}\right)\right]^{2 q\left(\mu_{j}-\mu_{j+1}\right)}}{\left[\Gamma\left(\mu_{j}-\mu_{j+1}+1\right)\right]^{2 q}} \int_{0}^{\pi}\left(\cos \theta_{j}\right)^{2 q\left(\mu_{j}-\mu_{j+1}\right)}\left(\sin \theta_{j}\right)^{2\left(q \mu_{j+1}+\alpha_{j}\right)} d \theta_{j} \\
= & 2 \pi \mathcal{N}_{l,\{\mu\}}^{2 q} \prod_{j=1}^{D-2} \frac{\left[2\left(\alpha_{j}+\mu_{j+1}\right)\right]^{2 q\left(\mu_{j}-\mu_{j+1}\right)}}{\left[\Gamma\left(\mu_{j}-\mu_{j+1}+1\right)\right]^{2 q}} B\left(q \mu_{j+1}+\frac{1}{2}+\alpha_{j}, q\left(\mu_{j}-\mu_{j+1}\right)+\frac{1}{2}\right) \\
= & (2 \pi)^{1+(1-D) q}\left(\prod_{j=1}^{D-2} \frac{\Gamma\left(q\left(\mu_{j}-\mu_{j+1}\right)+\frac{1}{2}\right)}{\Gamma\left(\mu_{j}-\mu_{j+1}+1\right)^{q}}\right)\left(\prod_{j=1}^{D-2}\left(\alpha_{j}+\mu_{j}\right)\left(\alpha_{j}+\mu_{j+1}\right)^{2\left(\mu_{j}-\mu_{j+1}\right)}\right)^{q} \\
& \times\left(\prod_{j=1}^{D-2} \frac{\Gamma\left(\alpha_{j}+q \mu_{j+1}+\frac{1}{2}\right)}{\Gamma\left(\alpha_{j}+q \mu_{j}+1\right)}\right)\left(\prod_{j=1}^{D-2} 4^{q\left(\alpha_{j}+\mu_{j}\right)} \frac{\Gamma\left(\alpha_{j}+\mu_{j+1}\right)^{2 q}}{\Gamma\left(2 \alpha_{j}+\mu_{j+1}+\mu_{j}\right)^{q}}\right) .
\end{aligned}
$$

Further algebraic manipulations, which are detailed in Appendix C, have allowed us to obtain that

$$
\begin{aligned}
\Lambda_{l,\{\mu\}}\left(\Omega_{D-1}\right) \sim & \left(2^{1-q} \frac{\Gamma\left(1+q \mu_{D-1}\right)}{\Gamma\left(1+\mu_{D-1}\right)^{q}} \tilde{\mathcal{M}}(D, q,\{\mu\})\right) \\
& \times\left(\tilde{\mathcal{E}}(D,\{\mu\})^{q} \pi^{\frac{D}{2}(1-q)} \frac{\Gamma\left(\frac{D}{2}+l\right)^{q}}{\Gamma\left(\frac{D}{2}+q l\right)}\right),
\end{aligned}
$$


where

$$
\tilde{\mathcal{M}}(D, q,\{\mu\}) \equiv 4^{q\left(l-\mu_{D-1}\right)} \pi^{1-\frac{D}{2}} \prod_{j=1}^{D-2} \frac{\Gamma\left(q\left(\mu_{j}-\mu_{j+1}\right)+\frac{1}{2}\right)}{\Gamma\left(\mu_{j}-\mu_{j+1}+1\right)^{q}}
$$

and

$$
\tilde{\mathcal{E}}(D,\{\mu\}) \equiv \prod_{j=1}^{D-2} \frac{\left(\alpha_{j}+\mu_{j+1}\right)^{2\left(\mu_{j}-\mu_{j+1}\right)}}{\left(2 \alpha_{j}+2 \mu_{j+1}\right)_{\mu_{j}-\mu_{j+1}}} \frac{1}{\left(\alpha_{j}+\mu_{j+1}\right) \mu_{j-\mu_{j+1}}} .
$$

Note that $\tilde{\mathcal{E}}=\tilde{\mathcal{M}}=1$ for any configuration with $\mu_{1}=\mu_{2}=\cdots=\mu_{D-1}$. Finally, as explained in Appendix $\mathrm{C}$, we have the following expression:

$$
\begin{aligned}
R_{q}\left[\mathcal{Y}_{l,\{\mu\}}\right] \sim & \frac{1}{1-q} \log \left(\frac{\Gamma\left(\frac{D}{2}+l\right)^{q}}{\Gamma\left(\frac{D}{2}+q l\right)}\right)+\frac{D}{2} \log \pi \\
& +\frac{1}{1-q} \log \left(\tilde{\mathcal{E}}(D,\{\mu\})^{q} \tilde{\mathcal{M}}(D, q,\{\mu\}) \frac{\Gamma\left(1+q \mu_{D-1}\right)}{\Gamma\left(1+\mu_{D-1}\right)^{q}} 2^{1-q}\right) \\
\sim & -\log \left(\Gamma\left(\frac{D}{2}\right)\right)+\frac{D}{2} \log \pi+\frac{1}{1-q} \log \left(\tilde{\mathcal{E}}(D,\{\mu\})^{q} \tilde{\mathcal{M}}(D, q,\{\mu\}) \frac{\Gamma\left(1+q \mu_{D-1}\right)}{\Gamma\left(1+\mu_{D-1}\right)^{q}} 2^{1-q}\right) \\
\sim & -\frac{D}{2} \log D+\frac{D}{2} \log (2 \pi e)+\frac{1}{2} \log D+\frac{1}{1-q} \log \left(\tilde{\mathcal{E}}(D,\{\mu\})^{q} \tilde{\mathcal{M}}(D, q,\{\mu\}) \frac{\Gamma\left(1+q \mu_{D-1}\right)}{\Gamma\left(1+\mu_{D-1}\right)^{q}}\left(\frac{\pi}{2}\right)^{\frac{q-1}{2}}\right)
\end{aligned}
$$

for the angular Rényi entropy of the generic hydrogenic state with hyperquantum numbers $(l,\{\mu\})$, which holds for every non-negative $q \neq 1$.

For completeness, we will determine this asymptotic behavior in a more complete manner for some physically relevant and experimentally accessible states such as the (ns) and circular ones, which are described by the hyperquantum numbers $(n, l=0,\{\mu\}=\{0\})$ and $(n, l=n-1,\{\mu\}=$ $\{n-1\})$, respectively. First we obtain from (34) the following values:

$$
\Lambda_{0,\{0\}}\left(\Omega_{D-1}\right) \sim 2 \pi \mathcal{N}_{0,\{0\}}^{2 q} \prod_{j=1}^{D-2} \frac{\Gamma\left(\frac{1}{2}\right) \Gamma\left(\alpha_{j}+\frac{1}{2}\right)}{\Gamma\left(\alpha_{j}+1\right)}=\left(\frac{2 \pi^{\frac{D}{2}}}{\Gamma\left(\frac{D}{2}\right)}\right)^{1-q}
$$

and

$$
\begin{aligned}
\Lambda_{n-1,\{n-1\}}\left(\Omega_{D-1}\right) & \sim 2 \pi \mathcal{N}_{n-, 1\{n-1\}}^{2 q} \prod_{j=1}^{D-2} \frac{\Gamma\left(\frac{1}{2}\right) \Gamma\left(\alpha_{j}+q(n-1) \frac{1}{2}\right)}{\Gamma\left(\alpha_{j}+q(n-1)+1\right)} \\
& =\left(2 \pi^{\frac{D}{2}}\right)^{1-q} \prod_{j=1}^{D-2}\left(\frac{\Gamma\left(\alpha_{j}+n\right)}{\Gamma\left(\alpha_{j}+n-\frac{1}{2}\right)}\right)^{q} \frac{\Gamma\left(\alpha_{j}+q(n-1)+\frac{1}{2}\right)}{\Gamma\left(\alpha_{j}+q(n-1)+1\right)} \\
& =\left(\frac{1}{2 \pi^{\frac{D}{2}}}\right)^{q-1} \frac{\left((n)_{\frac{D}{2}-1}\right)^{q}}{(1+q(n-1))_{\frac{D}{2}-1}},
\end{aligned}
$$

respectively. Note that $(x)_{a}=\frac{\Gamma(x+a)}{\Gamma(x)}$ is the well-known Pochhammer symbol. Then, from Eqs. (24), (39), and (40), we have that the angular part of the Rényi entropy at large $D$ is given by

$$
R_{q}\left[\mathcal{Y}_{0,\{0\}}\right] \sim \log \left(\frac{2 \pi^{\frac{D}{2}}}{\Gamma\left(\frac{D}{2}\right)}\right)
$$

and

$$
R_{q}\left[\mathcal{Y}_{n-1,\{n-1\}}\right] \sim \frac{1}{1-q} \log \left(\left(\frac{1}{2 \pi^{\frac{D}{2}}}\right)^{q-1} \frac{\left((n)_{\frac{D}{2}-1}\right)^{q}}{(1+q(n-1))_{\frac{D}{2}-1}}\right)
$$

for the $(n s)$ and circular states, respectively. Note that for very large $D$, the dominant term of the angular Rényi entropy of these two classes of physical states is the same, namely, $-\log \left(\Gamma\left(\frac{D}{2}\right)\right)+\frac{D}{2} \log \pi$. 
Moreover and most interesting, this is true for any hydrogenic state by taking into account the general expression (38). This observation allows us to conjecture the expression

$$
S\left[\mathcal{Y}_{l,\{\mu\}}\right] \sim-\log \left(\Gamma\left(\frac{D}{2}\right)\right)+\frac{D}{2} \log \pi
$$

for the large- $D$ behavior of the angular Shannon entropy of the hydrogenic states.

\section{Total position Rényi entropy}

To obtain the total Rényi entropy $R_{q}\left[\rho_{n, l,\{\mu\}}\right]$ in position space for a general $(n, l,\{\mu\})$-state, according to (22), we have to sum up the radial and angular contributions given by (31) and (38), respectively. Then, we obtain that

$$
\begin{aligned}
R_{q}\left[\rho_{n, l,\{\mu\}}\right] \sim & \log \left(\frac{D^{2 D}}{\Gamma\left(\frac{D}{2}\right)}\right)+D \log \left(\frac{q^{\frac{1}{q-1}} \sqrt{\pi}}{4 Z e}\right)+\frac{q\left(n-l-\frac{1}{2}\right)-\frac{1}{2}}{1-q} \log D \\
& +\frac{1}{1-q} \log \left(\tilde{\mathcal{E}}(D,\{\mu\})^{q} \tilde{\mathcal{M}}(D, q,\{\mu\}) \mathcal{F}(n, l, q) \frac{\Gamma\left(1+q \mu_{D-1}\right)}{\Gamma\left(1+\mu_{D-1}\right)^{q}}\left(\frac{\pi}{2}\right)^{\frac{q-1}{2}}\right) \\
\sim & \frac{3}{2} D \log D+D \log \left(\frac{q^{\frac{1}{q-1}}}{Z} \sqrt{\frac{\pi}{8 e}}\right)+\frac{q(n-l-1)}{1-q} \log D \\
& +\frac{1}{1-q} \log \left(\tilde{\mathcal{E}}(D,\{\mu\})^{q} \tilde{\mathcal{M}}(D, q,\{\mu\}) \mathcal{F}(n, l, q) \frac{\Gamma\left(1+q \mu_{D-1}\right)}{\Gamma\left(1+\mu_{D-1}\right)^{q}}\left(\frac{\pi}{2}\right)^{\frac{q-1}{2}}\right),
\end{aligned}
$$

which holds for every non-negative $q \neq 1$. Now, for completeness, we calculate this quantity in an explicit manner for the (ns) and circular states, which both of them include the ground state. For the $(n s)$ and circular states, we have the asymptotical expressions

$$
R_{q}\left[\rho_{n, 0,\{0\}}\right] \sim \log \left(\frac{D^{2 D}}{\Gamma\left(\frac{D}{2}\right)}\right)+D \log \left(\frac{q^{\frac{1}{q-1}} \sqrt{\pi}}{4 Z e}\right)+\frac{q(n-1)}{1-q} \log D+\frac{1}{1-q} \log (\mathcal{F}(n, 0, q))-\frac{1}{2} \log \frac{\pi}{2}
$$

(with $\left.\mathcal{F}(n, 0, q)=\frac{|q-1|^{2(n-1) q}}{(2 \pi)^{\frac{1}{2}(q-1)}[(n-1) !]^{q}}\right)$ and

$$
\begin{aligned}
R_{q}\left[\rho_{n, n-1,\{n-1\}}\right] \sim & \frac{1}{1-q} \log \left(\frac{\left((n)_{\frac{D}{2}-1}\right)^{q}}{(1+q(n-1))_{\frac{D}{2}-1}}\right)+2 D \log D \\
& +D \log \left(\frac{q^{\frac{1}{q-1}} \sqrt{\pi}}{4 Z e}\right)+\frac{1}{1-q} \log \left(\mathcal{F}(n, n-1, q) \frac{\Gamma(1+q(n-1))}{\Gamma(n)^{q}}\right)-\frac{1}{2} \log \frac{\pi}{2} \\
\sim & \log \left(\frac{D^{2 D}}{\Gamma\left(\frac{D}{2}\right)}\right)+D \log \left(\frac{q^{\frac{1}{q-1}} \sqrt{\pi}}{4 Z e}\right)+\frac{1}{1-q} \log \left(\frac{\Gamma(1+q(n-1))}{q^{2 q(n-1)} \Gamma(n)^{q}}\right)+2 n+\log 2-3,
\end{aligned}
$$

respectively. Most interesting, we realize that the large- $D$ behavior of the total Rényi entropy in the position space for the hydrogenic ground state $R_{q}\left[\rho_{1,0,\{0\}}\right]$ is given by the last expression above indicated.

Finally, from (44), one can conjecture that the Shannon entropy $S\left[\rho_{n, l,\{\mu\}}\right]$ in position space for a general $(n, l,\{\mu\})$-state is given by 


$$
\begin{aligned}
S\left[\rho_{n, l,\{\mu\}}\right] & \sim \log \left(\frac{D^{2 D}}{\Gamma\left(\frac{D}{2}\right)}\right)+D \log \left(\frac{\sqrt{\pi}}{4 Z}\right) \\
& \sim \frac{3}{2} D \log D+D \log \left(\frac{\sqrt{e \pi}}{\sqrt{8} Z}\right),
\end{aligned}
$$

but this is somehow risky because of the unknown $(q \rightarrow 1)$ behavior of the angular part, coming from the second line of Eq. (44).

\section{B. Rényi entropy in momentum space}

Let us now determine the momentum Rényi entropy of the probability density $\gamma_{n, l,\{\mu\}}(\vec{p})$ given by (18), which is defined as

$$
R_{q}\left[\gamma_{n, l,\{\mu\}}\right]=\frac{1}{1-q} \log W_{q}\left[\gamma_{n, l,\{\mu\}}\right] ; \quad 0<q<\infty, q \neq 1,
$$

where

$$
W_{q}\left[\gamma_{n, l,\{\mu\}}\right]=\int_{\mathbb{R}^{D}}\left[\gamma_{n, l,\{\mu\}}(\vec{p})\right]^{q} d \vec{p}
$$

denote the momentum entropic moments. Then, operating in a similar way as in position space, we obtain that

$$
R_{q}\left[\gamma_{n, l,\{\mu\}}\right]=R_{q}\left[\gamma_{n, l}\right]+R_{q}\left[\mathcal{Y}_{l,\{\mu\}}\right],
$$

where $R_{q}\left[\gamma_{n, l}\right]$ denotes the radial part,

$$
R_{q}\left[\gamma_{n, l}\right]=\frac{1}{1-q} \log \int_{0}^{\infty}\left[\mathcal{M}_{n, l}(p)\right]^{2 q} p^{D-1} d p
$$

and $R_{q}\left[\mathcal{Y}_{l,\{\mu\}}\right]$ denotes the angular part given by (24).

Since the angular entropy $R_{q}\left[\mathcal{Y}_{l,\{\mu\}}\right]$ has been already calculated and discussed, it only remains to determine at large $D$ the radial Rényi entropy $R_{q}\left[\gamma_{n, l}\right]$, given by (51), being all the hyperquantum numbers fixed.

\section{Radial momentum Rényi entropy}

To find the radial momentum Rényi entropy (51) at $D>>1$, we first rewrite for convenience the radial part of the wave function, $\mathcal{M}_{n, l}(p)$, given by (18), as

$$
\begin{array}{r}
\mathcal{M}_{n, l}(p)=\left(\frac{\eta}{Z}\right)^{\frac{D}{2}}(1+y)^{\frac{3}{2}}\left(\frac{1+y}{1-y}\right)^{\frac{D-2}{4}} \sqrt{w_{\alpha}(y)} \widehat{\mathcal{C}}_{n-l-1}^{(\alpha)}(y) \\
=\left(\frac{\eta}{Z}\right)^{\frac{D}{2}} A(n, l ; D)^{\frac{1}{2}}(1-y)^{\frac{l}{2}}(1+y)^{\frac{D+l+1}{2}} \mathcal{C}_{n-l-1}^{\left(l+\frac{D-1}{2}\right)}(y),
\end{array}
$$

with $y=\frac{1-\eta^{2} \tilde{p}^{2}}{1+\eta^{2} \tilde{p}^{2}}, \tilde{p}=p / Z, \alpha=l+\frac{D-1}{2}$, and $\widehat{\mathcal{C}}_{n-l-1}^{(\alpha)}(x)$ that denotes the orthonormal Gegenbauer polynomials with respect to the weight function $w_{\alpha}(x)=\left(1-x^{2}\right)^{\alpha-\frac{1}{2}}$, so that $\widehat{\mathcal{C}}_{n-l-1}^{\left(l+\frac{D-1}{2}\right)}(x)=A(n, l ; D)^{\frac{1}{2}}$ $\mathcal{C}_{n-l-1}^{\left(l+\frac{D-1}{2}\right)}(x)$, where the constant

$$
A(n, l ; D)=\frac{(n-l-1) !\left(n+\frac{D-3}{2}\right)\left[\Gamma\left(l+\frac{D-1}{2}\right)\right]^{2}}{2^{2-2 l-D} \pi \Gamma(n+l+D-2)} .
$$

The radial momentum Rényi entropy (51) together with (52) can be expressed as

$$
R_{q}\left[\mathcal{M}_{n, l}\right]=-D \log \frac{\eta}{Z}+\frac{1}{1-q}\left\{q \log A(n, l ; D)+\log I_{n, l}(q, D)\right\}
$$

and the Rényi-like functional of Gegenbauer polynomials is as follows:

$$
I_{n, l}(q, D)=\int_{-1}^{1}(1-y)^{l q-1+\frac{D}{2}}(1+y)^{(l+1) q-1+\left(q-\frac{1}{2}\right) D}\left[\mathcal{C}_{n-l-1}^{\left(l+\frac{D-1}{2}\right)}(y)\right]^{2 q} d y .
$$


It only remains to find the large- $D$ behavior of the two terms in (54) when $(n, l, q)$ are fixed. The asymptotic estimate of $A(n, l ; D)$ turns out to be given by

$$
A(n, l ; D) \sim \frac{\Gamma(n-l)}{\sqrt{2 \pi}} D^{l-n+\frac{3}{2}} .
$$

On the other hand, the behavior of $I_{n, l}(q, D)$ at large $D$ can be obtained from Theorem 2 (see Appendix B) by studying the large- $\alpha$ behavior of the integral $J_{2}\left(a, b, c, d, \kappa, m^{\prime} ; \alpha\right)$ with the parameters $a=l(q-1)-\frac{1}{2}, b=l(1-q)+2 q-\frac{3}{2}, c=1, d=2 q-1, \kappa=2 q, m^{\prime}=n-l-1$ and $\alpha=l+\frac{D-1}{2}$. Note that the condition $c<d$ of the theorem provokes that $q>1$. We have found that

$$
\begin{array}{r}
I_{n, l}(q, D) \sim\left(\frac{(2 q-1)^{2 q-1}}{q^{2 q}}\right)^{\frac{D}{2}}\left(\frac{\Gamma\left(\frac{D}{2}+n-\frac{3}{2}\right)}{\Gamma\left(\frac{D}{2}+l-\frac{1}{2}\right)}\right)^{2 q}(D+2 l-1)^{-\frac{1}{2}} Q_{0}(n, l, q) \\
\sim\left(\frac{(2 q-1)^{2 q-1}}{q^{2 q}}\right)^{\frac{D}{2}}\left(\frac{D}{2}\right)^{2 q(n-l-1)}(D+2 l-1)^{-\frac{1}{2}} Q_{0}(n, l, q) \\
\sim\left(\frac{(2 q-1)^{2 q-1}}{q^{2 q}}\right)^{\frac{D}{2}} D^{2 q(n-l-1)-\frac{1}{2}} \frac{Q_{0}(n, l, q)}{4^{q(n-l-1)}}
\end{array}
$$

for $q>1$ and where $Q_{0}(q)$ is given by

$$
Q_{0}(q, n, l)=\frac{\sqrt{2 \pi} 4^{q(n-l-1)}}{\Gamma(n-l)^{2 q}} \frac{(2 q-1)^{q(l+1)-\frac{1}{2}}(q-1)^{2 q(n-l-1)}}{q^{q(2 n-1)-\frac{1}{2}}} .
$$

Using (56) and (57), we obtain the following behavior at large $D$ for the radial part of the momentum Rényi entropy:

$$
\begin{aligned}
R_{q}\left[\mathcal{M}_{n, l}\right] \sim & -D \log \left(\frac{\eta}{Z}\right)+\frac{D}{1-q} \log \sqrt{\frac{(2 q-1)^{2 q-1}}{q^{2 q}}}+\frac{q\left(n-l-\frac{1}{2}\right)-\frac{1}{2}}{1-q} \log D \\
& +\frac{1}{1-q} \log \bar{Q}_{0}(q, n, l) \\
\sim & -D \log \left(\frac{D}{2 Z}\right)+\frac{D}{1-q} \log \sqrt{\frac{(2 q-1)^{2 q-1}}{q^{2 q}}}+\frac{q\left(n-l-\frac{1}{2}\right)-\frac{1}{2}}{1-q} \log D \\
& +\frac{1}{1-q} \log \bar{Q}_{0}(q, n, l)
\end{aligned}
$$

with $\bar{Q}_{0}(q, n, l)=\frac{\Gamma(n-l)^{q}}{(2 \pi)^{\frac{q}{2}} 4^{q(n-l-1)}} Q_{0}(q, n, l)=\frac{(2 \pi)^{\frac{1-q}{2}}}{\Gamma(n-l)^{q}} \frac{(2 q-1)^{q(l+1)-\frac{1}{2}}(q-1)^{2 q(n-l-1)}}{q^{q(2 n-1)-\frac{1}{2}}}$ (where we have used in the second expression that $\eta=n+\frac{D-3}{2} \sim \frac{D}{2}$ for fixed $\left.n\right)$ for a general $(n, l)$-state. Note that in the limit $q \rightarrow 1$, this expression suggests that the behavior of the radial Shannon entropy in the momentum space can be conjectured at large $D$ as

$$
S\left[\mathcal{M}_{n, l}\right] \sim-D \log \left(\frac{D}{2 Z}\right) .
$$

Then, according to Eq. (50), to fix the large $D$-behavior of the total Rényi entropy $R_{p}\left[\gamma_{n, l,\{\mu\}}\right]$, it only remains the evaluation of the corresponding behavior of the angular part $R_{p}\left[\mathcal{Y}_{l,\{\mu\}}\right]$, which was found in (38).

\section{Total momentum Rényi entropy}

To obtain the total Rényi entropy in momentum space for a general $(n, l,\{\mu\})$-state of a largedimensional hydrogenic system, we have to sum up the radial and angular contributions, given by 
(50), and then to take into account the final expressions (59) and (38) for these contributions. Then, it follows the expression

$$
\begin{aligned}
R_{q}\left[\gamma_{n, l,\{\mu\}}\right] \sim & -\log \left(\frac{\eta^{D} \Gamma\left(\frac{D}{2}\right)}{Z^{D}}\right)+D \log \left(\sqrt{\pi}\left(\frac{(2 q-1)^{2 q-1}}{q^{2 q}}\right)^{\frac{1}{2-2 q}}\right) \\
& +\frac{q\left(n-l-\frac{1}{2}\right)-\frac{1}{2}}{1-q} \log D+\frac{1}{1-q} \log \left(\tilde{\mathcal{E}}(D,\{\mu\})^{q} \tilde{\mathcal{M}}(D, q,\{\mu\}) \bar{Q}_{0}(q, n, l) \pi^{\frac{q-1}{2}}\right) \\
\sim & -\frac{3}{2} D \log D+D \log \left(Z \sqrt{8 e \pi}\left(\frac{(2 q-1)^{2 q-1}}{q^{2 q}}\right)^{\frac{1}{2-2 q}}\right) \\
& +\frac{q(n-l-1)}{1-q} \log D+\frac{1}{1-q} \log \left(\tilde{\mathcal{E}}(D,\{\mu\})^{q} \tilde{\mathcal{M}}(D, q,\{\mu\}) \bar{Q}_{0}(q, n, l) \pi^{\frac{q-1}{2}}\right)
\end{aligned}
$$

(with $q \neq 1$ and $\eta \sim \frac{D}{2}$ ) for the large- $D$ behavior of the total momentum Rényi entropy of the generic hydrogenic state $(n, l,\{\mu\})$, where the symbols $\tilde{\mathcal{M}}(D, q,\{\mu\})$ and $\tilde{\mathcal{E}}(D,\{\mu\})$ are defined in Eqs. (36) and (37), respectively. For completeness and illustration, let us give in a more complete manner the behavior of this quantity at $D>>1$ for some particular quantum states such as the $(n s)$ and circular states. For the (ns)-states, we found

$$
\begin{aligned}
R_{q}\left[\gamma_{n, 0,\{0\}}\right] \sim & -\frac{3}{2} D \log D+D \log \left(Z \sqrt{8 e \pi}\left(\frac{(2 q-1)^{2 q-1}}{q^{2 q}}\right)^{\frac{1}{2-2 q}}\right) \\
& +\frac{q(n-1)}{1-q} \log D+\frac{1}{1-q} \log \left(\bar{Q}_{0}(q, n, 0) \pi^{\frac{q-1}{2}}\right)
\end{aligned}
$$

with

$$
\bar{Q}_{0}(q, n, 0)=\frac{(2 \pi)^{\frac{1-q}{2}}}{\Gamma(n)^{q}} \frac{(2 q-1)^{q-\frac{1}{2}}(q-1)^{2 q(n-1)}}{q^{q(2 n-1)-\frac{1}{2}}} .
$$

And for the circular states, we obtained the following large- $D$ behavior:

$$
\begin{aligned}
R_{q}\left[\gamma_{n, n-1,\{n-1\}}\right] \sim & -\frac{3}{2} D \log D+D \log \left(Z \sqrt{8 e \pi}\left(\frac{(2 q-1)^{2 q-1}}{q^{2 q}}\right)^{\frac{1}{2-2 q}}\right) \\
& +\frac{1}{1-q} \log \left(\bar{Q}_{0}(q, n, n-1) \pi^{\frac{q-1}{2}}\right)
\end{aligned}
$$

with

$$
\bar{Q}_{0}(q, n, n-1)=(2 \pi)^{\frac{1-q}{2}} \frac{(2 q-1)^{q n-\frac{1}{2}}}{q^{q(2 n-1)-\frac{1}{2}}} .
$$

Note now that either from (62) or from (64) with $n=1$, we have the following large- $D$ behavior:

$$
\begin{aligned}
R_{q}\left[\gamma_{1,0,\{0\}}\right] \sim & -\frac{3}{2} D \log D+D \log \left(Z \sqrt{8 e \pi}\left(\frac{(2 q-1)^{2 q-1}}{q^{2 q}}\right)^{\frac{1}{2-2 q}}\right) \\
& +\frac{1}{1-q} \log \left(\bar{Q}_{0}(q, 1,0) \pi^{\frac{q-1}{2}}\right)
\end{aligned}
$$

with $q \neq 1$ and $\bar{Q}_{0}(q, 1,0)=(2 \pi)^{\frac{1-q}{2}}\left(2-\frac{1}{q}\right)^{q-\frac{1}{2}}$ for the total momentum Rényi entropy of the ground hydrogenic state.

Finally, from (61), one can conjecture that the Shannon entropy $S\left[\rho_{n, l,\{\mu\}}\right]$ in momentum space for a general $(n, l,\{\mu\})$-state is given by

$$
S\left[\gamma_{n, l,\{\mu\}}\right] \sim-\frac{3}{2} D \log D+D \log (Z \sqrt{8 e \pi})
$$

in the limiting case $q \rightarrow 1$. 


\section{Position-momentum Rényi-entropy-based uncertainty sum}

From Eqs. (44) and (61), we can obtain the dominant term for the joint position-momentum Rényientropy-based uncertainty sum of a large-dimensional hydrogenic system for a pair of parameters $p$ and $q$ which fulfill the Holder conjugacy relation $\frac{1}{p}+\frac{1}{q}=2$. We found that

$$
\begin{aligned}
R_{q}\left[\rho_{n, l,\{\mu\}}\right]+R_{p}\left[\gamma_{n, l,\{\mu\}}\right] & \sim D \log \left[\pi\left(\frac{(2 p-1)^{(2 p-1)}}{p^{2 p}}\right)^{\frac{1}{2-2 p}} q^{\frac{1}{q-1}}\right] \\
& =D \log \left[2 \pi(2 p)^{\frac{1}{2 p-2}}(2 q)^{\frac{1}{2 q-2}}\right], \quad q \neq 1,
\end{aligned}
$$

for all $(n, l,\{\mu\})$-states, which saturates the known position-momentum Rényi-entropy-based uncertainty relation. ${ }^{38-40}$ Note that out of the so-called conjugacy curve (i.e., for arbitrary positive pairs of values of $p$ and $q$ ), there is a dependence at second order on the quantum numbers $n$ and $l$; this dependence just disappears onto the conjugation line. A more detailed study of this behaviour out of the conjugacy curve remains as an open problem. Finally, the conjectured expressions for Shannon entropy in both spaces (47) and (67) allows one to write

$$
S\left[\rho_{n, l,\{\mu\}}\right]+S\left[\gamma_{n, l,\{\mu\}}\right] \sim D \log [2 \pi e],
$$

which saturates the general Bialynicki-Birula-Mycielski entropic relation. ${ }^{35,38}$

\section{CONCLUSIONS}

In this work, we have determined the large- $D$ behavior of the position and momentum Rényi entropies of the $D$-dimensional hydrogenic states at large $D$ in terms of the state's hyperquantum numbers and the nuclear charge $Z$ of the system. We have used a recent constructive methodology which allows for the calculation of some Rényi-like integral functionals of Laguerre $\mathcal{L}_{k}^{(\alpha)}(x)$ and Gegenbauer $\mathcal{C}_{k}^{\left(\alpha^{\prime \prime}\right)}(x)$ polynomials with a fixed degree $k$ and large values of the parameters $\alpha$ and $\alpha^{\prime \prime}$. This has been possible because the hydrogenic states are controlled by the Laguerre and Gegenbauer polynomials in position space and by the Gegenbauer polynomials in momentum space, keeping in mind that the hyperspherical harmonics (which determine the angular part of the wave functions in the two conjugated spaces) can be expressed in terms of the latter polynomials. Then, simple expressions of these quantities for some specific classes of hydrogenic states ( $n s$ and circular states), which include the ground state, are given. Moreover, as a byproduct, our results reach the saturation of the known position-momentum Rényi-entropy-based uncertainty relations. To this respect, we should keep in mind that we are assuming that the dimensionality is very large and the hyperquantum numbers are small. The exceptional case when both dimensionality and hyperquantum numbers are simultaneously large has not yet been explored; in particular, we cannot assure saturation.

We should highlight that to find the Shannon entropies of the large-dimensional hydrogenic systems have not yet been possible with the present methodology although the dominant term has been conjectured. A rigorous proof remains open.

Finally, let us mention that it would be very relevant for many quantum-mechanical problems other than the hydrogenic ones (e.g., the harmonic systems) the determination of the behavior of integral functionals of Rényi and Shannon types for hypergeometric polynomials other than the Laguerre and Gegenbauer ones at large values of the polynomials' parameters and fixed degrees. This is yet another open problem for the future.

\section{ACKNOWLEDGMENTS}

This work has been partially supported by the Project Nos. FQM-7276 and FQM-207 of the Junta de Andalucía and the MINECO (Ministerio de Economia y Competitividad)-FEDER (European Regional Development Fund) Grant Nos. FIS2014- 54497P and FIS2014-59311-P. I. V. Toranzo acknowledges the support of ME under the program FPU. N. M. Temme acknowledges financial support from Ministerio de Ciencia e Innovación, Project No. MTM2012-11686, and thanks CWI, 
Amsterdam, for scientific support. Finally, some mathematical discussions with N. Sobrino-Coll are acknowledged.

\section{APPENDIX A: RÉNYI-LIKE FUNCTIONALS OF LAGUERRE POLYNOMIALS WITH LARGE PARAMETERS}

In this appendix, the asymptotics ( $\alpha \rightarrow \infty$ ) of some Rényi-like functionals of the Laguerre polynomials is given by means of the following theorem which has been recently found ${ }^{43}$ (see also Refs. 44 and 45). Herein, the involved parameters are just algebraic numbers without any quantum interpretation.

Theorem 1. The Rényi-like functional of the Laguerre polynomials $\mathcal{L}_{m}^{(\alpha)}(x)$ given by

$$
J_{1}(\sigma, \lambda, \kappa, m ; \alpha)=\int_{0}^{\infty} x^{\alpha+\sigma-1} e^{-\lambda x}\left|\mathcal{L}_{m}^{(\alpha)}(x)\right|^{\kappa} d x
$$

(with $\sigma$ real, $0<\lambda \neq 1, \kappa>0$ ) has the following $(\alpha \rightarrow \infty)$-asymptotic behavior:

$$
J_{1}(\sigma, \lambda, \kappa, m ; \alpha) \sim \alpha^{\alpha+\sigma} e^{-\alpha} \lambda^{-\alpha-\sigma-\kappa m}|\lambda-1|^{\kappa m} \sqrt{\frac{2 \pi}{\alpha}} \frac{\alpha^{\kappa m}}{(m !)^{\kappa}} \sum_{j=0}^{\infty} \frac{D_{j}}{\alpha^{j}},
$$

with the first coefficients $D_{0}=1$ and

$$
\begin{aligned}
D_{1}= & \frac{1}{12(\lambda-1)^{2}}\left(1-12 \kappa m \sigma \lambda+6 \sigma^{2} \lambda^{2}-12 \sigma^{2} \lambda-6 \sigma \lambda^{2}+12 \sigma \lambda+\right. \\
& 6 \kappa^{2} m^{2}+12 \kappa m \sigma-12 \kappa m^{2} \lambda-12 \kappa m \lambda+6 \kappa m \lambda^{2}+ \\
& \left.6 \kappa m^{2} \lambda^{2}+\lambda^{2}+6 \sigma^{2}-2 \lambda-6 \sigma+6 \kappa m^{2}\right) .
\end{aligned}
$$

For the knowledge of the remaining coefficients and further details about the theorem, see Ref. 43.

\section{APPENDIX B: RÉNYI-LIKE FUNCTIONALS OF GEGENBAUER POLYNOMIALS WITH LARGE PARAMETERS}

In this appendix, the asymptotics $(\alpha \rightarrow \infty)$ of some Rényi-like functionals of Gegenbauer polynomials is given by means of the following theorem which has been recently found ${ }^{43}$ (see also Refs. 44 and 45). Herein, the involved parameters are just algebraic numbers without any quantum interpretation.

Theorem 2. Let $a, b, c, d$, and $\kappa$ be positive real numbers, $c<d$, and m a positive natural number. Then, the Rényi-like functional of the Gegenbauer polynomials $\mathcal{C}_{m}^{(\alpha)}(x)$ given by

$$
J_{2}(a, b, c, d, \kappa, m ; \alpha)=\int_{-1}^{1}(1-x)^{c \alpha+a}(1+x)^{d \alpha+b}\left|\mathcal{C}_{m}^{(\alpha)}(x)\right|^{\kappa} d x
$$

has the following asymptotics:

$$
J_{2}(a, b, c, d, \kappa, m ; \alpha) \sim e^{-\alpha \phi\left(x_{m}\right)} \sqrt{\frac{2 \pi}{\alpha}} \frac{2^{\kappa m}\left((\alpha)_{m}\right)^{\kappa}}{(m !)^{\kappa}} \sum_{k=0}^{\infty} \frac{D_{k}}{\alpha^{k}}, \quad \alpha \rightarrow \infty,
$$

where the coefficients $D_{k}$ do not depend on $\alpha$. The first coefficient is given by

$$
D_{0}=a_{1}\left(\frac{2 c}{c+d}\right)^{a}\left(\frac{2 d}{c+d}\right)^{b}\left(\frac{d-c}{c+d}\right)^{\kappa m}
$$

and the symbols $x_{m}=(d-c) /(d+c), \phi\left(x_{m}\right)=-c \log \frac{2 c}{c+d}-d \log \frac{2 d}{c+d}$, and $a_{1}=2 \sqrt{\frac{c d}{(c+d)^{3}}}$. 
Moreover, if $c=d$, the corresponding Rényi-like functional

$$
J_{2}(a, b, c, \kappa, m ; \alpha)=\int_{-1}^{1}(1-x)^{a}(1+x)^{b} e^{-\alpha \phi(x)}\left|\mathcal{C}_{m}^{(\alpha)}(x)\right|^{\kappa} d x
$$

has the asymptotic behavior

$$
J_{2}(a, b, c, \kappa, m ; \alpha) \sim \sqrt{\frac{\pi}{\alpha c}} \frac{(2 \alpha)^{m}}{m !}, \quad \alpha \rightarrow \infty .
$$

Finally, the asymptotics of the Rényi-like functional with $c>d$ follows from the one with $c<d$ by interchanging $a$ and $b$ and $c$ and $d$. The case $c>d$ is useful for the determination of the Rényi entropy of the large dimensional hydrogenic states in momentum space with $q<1$. For further details of the theorem, see Ref. 43.

\section{APPENDIX C: LARGE-D BEHAVIOR OF THE ANGULAR RÉNYI FACTOR $\Lambda_{l,\{\mu\}}\left(\Omega_{D-1}\right)$}

Here we gather the necessary steps to obtain the final asymptotical expression (35) for the angular Rényi factor $\Lambda_{l,\{\mu\}}\left(\Omega_{D-1}\right)$ from Eq. (34). First of all, we have that since $\alpha_{j+1}=\alpha_{j}-\frac{1}{2}$, one has that

$$
\prod_{j=1}^{D-2} \frac{\Gamma\left(\alpha_{j}+q \mu_{j+1}+\frac{1}{2}\right)}{\Gamma\left(\alpha_{j}+q \mu_{j}+1\right)}=\frac{\Gamma\left(\alpha_{D-2}+q \mu_{D-1}+\frac{1}{2}\right)}{\Gamma\left(\alpha_{1}+q \mu_{1}+1\right)}=\frac{\Gamma\left(1+q \mu_{D-1}\right)}{\Gamma\left(\frac{D}{2}+q l\right)} .
$$

Then, we can express the product

$$
\prod_{j=1}^{D-2} 4^{q\left(\alpha_{j}+\mu_{j}\right)} \frac{\Gamma\left(\alpha_{j}+\mu_{j+1}\right)^{2 q}}{\Gamma\left(2 \alpha_{j}+\mu_{j+1}+\mu_{j}\right)^{q}}=\prod_{j=1}^{D-2} 4^{q\left(\alpha_{j}+\mu_{j}\right)} \frac{\Gamma\left(\alpha_{j}+\mu_{j+1}\right)^{2 q}}{\Gamma\left(2 \alpha_{j}+2 \mu_{j+1}\right)^{q}} \prod_{j=1}^{D-2} \frac{\Gamma\left(2 \alpha_{j}+2 \mu_{j+1}\right)^{q}}{\Gamma\left(2 \alpha_{j}+\mu_{j+1}+\mu_{j}\right)^{q}},
$$

whose first factor can be rewritten as

$$
\begin{aligned}
& \prod_{j=1}^{D-2} 4^{q\left(\alpha_{j}+\mu_{j}\right)} \frac{\Gamma\left(\alpha_{j}+\mu_{j+1}\right)^{2 q}}{\Gamma\left(2 \alpha_{j}+2 \mu_{j+1}\right)^{q}}=\prod_{j=1}^{D-2} 4^{q\left(\mu_{j}-\mu_{j+1}\right)}(2 \sqrt{\pi})^{q} \frac{\Gamma\left(\alpha_{j}+\mu_{j+1}+1\right)^{q}}{\Gamma\left(\alpha_{j}+\mu_{j+1}+\frac{1}{2}\right)^{q}}\left(\alpha_{j}+\mu_{j+1}\right)^{-q} \\
= & 4^{q\left(l-\mu_{D-1}\right)}(2 \sqrt{\pi})^{q(D-2)} \frac{\Gamma\left(\frac{D}{2}+l\right)^{q}}{\Gamma\left(1+\mu_{D-1}\right)^{q}} \prod_{j=1}^{D-2} \frac{\Gamma\left(\alpha_{j}+\mu_{j+1}+1\right)^{q}}{\Gamma\left(\alpha_{j}+\mu_{j}+1\right)^{q}}\left(\alpha_{j}+\mu_{j+1}\right)^{-q} .
\end{aligned}
$$

Taking into account these previous observations, Eq. (34) becomes

$$
\begin{aligned}
\Lambda_{l,\{\mu\}}\left(\Omega_{D-1}\right) \sim 2^{1-q} 4^{q\left(l-\mu_{D-1}\right)} \pi^{1-q \frac{D}{2}} \mathcal{M}(D, q,\{\mu\})(\mathcal{A}(D,\{\mu\}))^{q} \times \\
\frac{\Gamma\left(\frac{D}{2}+l\right)^{q}}{\Gamma\left(\frac{D}{2}+q l\right)} \frac{\Gamma\left(1+q \mu_{D-1}\right)}{\Gamma\left(1+\mu_{D-1}\right)^{q}} \prod_{j=1}^{D-2} \frac{\Gamma\left(2 \alpha_{j}+2 \mu_{j+1}\right)^{q}}{\Gamma\left(2 \alpha_{j}+\mu_{j+1}+\mu_{j}\right)^{q}} \frac{\Gamma\left(\alpha_{j}+\mu_{j+1}+1\right)^{q}}{\Gamma\left(\alpha_{j}+\mu_{j}+1\right)^{q}},
\end{aligned}
$$

where

$$
\mathcal{M}(D, q,\{\mu\}) \equiv \prod_{j=1}^{D-2} \frac{\Gamma\left(q\left(\mu_{j}-\mu_{j+1}\right)+\frac{1}{2}\right)}{\Gamma\left(\mu_{j}-\mu_{j+1}+1\right)^{q}}
$$

and

$$
\mathcal{A}(D,\{\mu\}) \equiv \prod_{j=1}^{D-2}\left(\alpha_{j}+\mu_{j}\right)\left(\alpha_{j}+\mu_{j+1}\right)^{2\left(\mu_{j}-\mu_{j+1}\right)-1} .
$$

Now, for convenience, we introduce the notation

$$
\begin{aligned}
\tilde{\mathcal{E}}(D,\{\mu\}) & \equiv \mathcal{A}(D,\{\mu\}) \times \prod_{j=1}^{D-2} \frac{\left(2 \alpha_{j}+2 \mu_{j+1}\right)_{\mu_{j}-\mu_{j+1}}^{-1}}{\left(\alpha_{j}+\mu_{j+1}+1\right)_{\mu_{j}-\mu_{j+1}}} \\
& =\prod_{j=1}^{D-2} \frac{\left(\alpha_{j}+\mu_{j+1}\right)^{2\left(\mu_{j}-\mu_{j+1}\right)}}{\left(2 \alpha_{j}+2 \mu_{j+1}\right)_{\mu_{j}-\mu_{j+1}}} \frac{1}{\left(\alpha_{j}+\mu_{j+1}\right)_{\mu_{j}-\mu_{j+1}}}
\end{aligned}
$$


so that we have

$$
\begin{aligned}
\Lambda_{l,\{\mu\}}\left(\Omega_{D-1}\right) \sim & \left(\pi 2^{1-q+2 q\left(l-\mu_{D-1}\right)} \frac{\Gamma\left(1+q \mu_{D-1}\right)}{\Gamma\left(1+\mu_{D-1}\right)^{q}}\right) \\
& \times\left(\mathcal{M}(D, q,\{\mu\}) \frac{\tilde{\mathcal{E}}(D,\{\mu\})^{q}}{\pi^{q \frac{D}{2}}} \frac{\Gamma\left(\frac{D}{2}+l\right)^{q}}{\Gamma\left(\frac{D}{2}+q l\right)}\right),
\end{aligned}
$$

where the dominant factor is $\frac{\Gamma\left(\frac{D}{2}+l\right)^{q}}{\Gamma\left(\frac{D}{2}+q l\right)} \sim \Gamma\left(\frac{D}{2}\right)^{q-1}$. Moreover we can still simplify this expression by the use of the notation $\tilde{\mathcal{M}}$,

$$
\tilde{\mathcal{M}}(D, q,\{\mu\}) \equiv 4^{q\left(l-\mu_{D-1}\right)} \pi^{1-\frac{D}{2}} \mathcal{M}(D, q,\{\mu\})
$$

to have $\tilde{\mathcal{M}} \equiv 1$ for all configurations with $\mu_{1}=\mu_{2}=\cdots=\mu_{D-1}$. Thus, we can finally obtain the searched expression (35), namely,

$$
\begin{aligned}
\Lambda_{l,\{\mu\}}\left(\Omega_{D-1}\right) \sim & \left(2^{1-q} \frac{\Gamma\left(1+q \mu_{D-1}\right)}{\Gamma\left(1+\mu_{D-1}\right)^{q}}\right) \\
& \times\left(\tilde{\mathcal{M}}(D, q,\{\mu\}) \tilde{\mathcal{E}}(D,\{\mu\})^{q} \pi^{\frac{D}{2}(1-q)} \frac{\Gamma\left(\frac{D}{2}+l\right)^{q}}{\Gamma\left(\frac{D}{2}+q l\right)}\right) .
\end{aligned}
$$

This expression together with (24) allow us to determine the dominant term of the angular Rényi entropy for fixed $l$ as

$$
R_{q}\left[\mathcal{Y}_{l,\{\mu\}}\right] \sim-\log \left(\Gamma\left(\frac{D}{2}\right)\right)+\frac{D}{2} \log \pi+\frac{1}{1-q} \log \left(\tilde{\mathcal{E}}(D,\{\mu\})^{q} \tilde{\mathcal{M}}(D, q,\{\mu\})\right)
$$

(where the third term vanishes for $\mu_{1}=\mu_{2}=\cdots=\mu_{D-1}$ ) which corresponds to expression (38) with the values of $\tilde{\mathcal{M}}(D, q,\{\mu\})$ and $\tilde{\mathcal{E}}(D,\{\mu\})$ given above.

${ }^{1}$ E. Witten, "Quarks, atoms, and the 1/N expansion," Phys. Today 33(7), 38-43 (1980).

${ }^{2}$ A. Chatterjee, "Large- $N$ expansions in quantum mechanics, atomic physics and some $O(N)$ invariant systems," Phys. Rep. 186, 249 (1990).

${ }^{3}$ Dimensional Scaling in Chemical Physics, edited by D. R. Herschbach, J. Avery, and O. Goscinski (Kluwer Academic Publishers, London, 1993).

${ }^{4}$ C. T. Tsipis, V. S. Popov, D. R. Herschbach, and J. S. Avery, New Methods in Quantum Theory (Kluwer Academic Publishers, Dordrecht, 1996)

${ }^{5}$ D. R. Herschbach, "Dimensional interpolation for two-electron atoms," J. Chem. Phys. 84, 838 (1986).

${ }^{6}$ D. R. Herschbach, "Fifty years in physical chemistry: Homage to mentors, methods, and molecules," Annu. Rev. Phys. Chem. 51, 1-39 (2000).

${ }^{7}$ L. G. Yaffe, "Large $N$ limits as classical mechanics," Rev. Mod. Phys. 54, 407 (1982).

${ }^{8}$ L. G. Yaffe, "Large- $N$ quantum mechanics and classical limits," Phys. Today 36(8), 50 (1983).

${ }^{9}$ D. R. Herschbach, "Dimensional scaling and renormalization," Int. J. Quantum Chem. 57, 295 (1996).

${ }^{10}$ R. J. Yáñez, W. Van Assche, and J. S. Dehesa, "Position and momentum information entropies of the $D$-dimensional harmonic oscillator and hydrogen atom," Phys. Rev. A 50(4), 3065 (1994).

${ }^{11}$ M. M. Nieto, "Hydrogen atom and relativistic pi-mesic atom in N-space dimensions," Am. J. Phys. 47(12), 1067 (1979).

12 J. S. Dehesa, S. López-Rosa, A. Martínez-Finkelshtein, and R. J. Yáñez, "Information theory of $D$-dimensional hydrogenic systems: Application to circular and Rydberg states," Int. J. Quantum Chem. 110, 1529-1548 (2010).

${ }^{13}$ S. Pasternack, "On the mean value of $r^{s}$ for Keplerian systems," Proc. Natl. Acad. Sci. U. S. A. 23, 91 (1937).

${ }^{14}$ A. Ray, K. Mahata, and P. P. Ray, "Moments of probability distribution, wave functions, and their derivatives at the origin of $N$-dimensional central potentials," Am. J. Phys. 56, 462 (1988).

${ }^{15}$ G. W. F. Drake and R. A. Swainson, "Expectation values of $r^{P}$ for arbitrary hydrogenic states," Phys. Rev. A 42, 1123 (1990).

${ }^{16} \mathrm{D}$. Andrae, "Recursive evaluation of expectation values for arbitrary states of the relativistic one-electron atom," J. Phys. B: At., Mol. Opt. Phys. 30, 4435 (1997).

${ }^{17}$ V. F. Tarasov, "Exact numerical values of diagonal matrix elements $\left\langle r^{k}\right\rangle_{n l}$, AS $n \leq 8$ and $-7 \leq k \leq 4$, and the symmetry of Appell's function $F_{2}(1,1)$," Int. J. Mod. Phys. B 18, 3177-3184 (2004).

${ }^{18}$ A. Guerrero, P. Sánchez-Moreno, and J. S. Dehesa, "Upper bounds on quantum uncertainty products and complexity measures," Phys. Rev. A 84, 042105 (2011).

${ }^{19}$ J. D. Hey, "On the momentum representation of hydrogenic wave functions: Some properties and an application,” Am. J. Phys. 61, 28 (1993).

${ }^{20}$ W. van Assche, R. J. Yáñez, R. González-Férez, and J. S. Dehesa, "Functionals of Gegenbauer polynomials and $D$ dimensional hydrogenic momentum expectation values," J. Math. Phys. 41, 6600 (2000).

${ }^{21}$ A. I. Aptekarev, J. S. Dehesa, A. Martínez-Finkelshtein, and R. J. Yáñez, "Quantum expectation values of $D$-dimensional Rydberg hydrogenic states by use of Laguerre and Gegenbauer asymptotics,” J. Phys. A: Math. Theor. 43, 145204 (2010). 
${ }^{22}$ I. V. Toranzo, A. Martínez-Finkelshtein, and J. S. Dehesa, "Heisenberg-like uncertainty measures for $D$-dimensional hydrogenic systems at large D,” J. Math. Phys. 57, 082109 (2016).

${ }^{23}$ V. Buyarov, J. S. Dehesa, A. Martínez-Finkelshtein, and J. Sánchez-Lara, "Computation of the entropy of polynomials orthogonal on an interval," SIAM J. Sci. Comput. 26(2), 488-509 (2004).

${ }^{24}$ I. V. Toranzo and J. S. Dehesa, "Rényi, Shannon and Tsallis entropies of Rydberg hydrogenic systems," Europhys. Lett. 113, 48003 (2016).

${ }^{25}$ I. V. Toranzo, D. Puertas-Centeno, and J. S. Dehesa, "Entropic properties of $D$-dimensional Rydberg systems," Physica A 462, 1197 (2016).

${ }^{26}$ A. I. Aptekarev, D. N. Tulyakov, I. V. Toranzo, and J. S. Dehesa, "Rényi entropies of the highly-excited states of multidimensional harmonic oscillators by use of strong Laguerre asymptotics," Eur. Phys. J. B 89, 85 (2016).

${ }^{27}$ A. Rényi, "On measures of entropy and information," in Proceedings of the 4th Berkeley Symposium on Mathematical Statistics and Probability, edited by J. Neyman (University of California Press, Berkeley, 1961), Vol. 1, pp. 547-561.

${ }^{28}$ C. E. Shannon, "A mathematical theory of communication," Bell Syst. Tech. J. 27(3), 379 (1948).

${ }^{29}$ J. Aczel and Z. Daroczy, On Measures of Information and Their Characterizations (Academic Press, New York, 1975).

${ }^{30}$ E. Romera, J. C. Angulo, and J. S. Dehesa, “The Hausdorff entropic moment problem,” J. Math. Phys. 42, 2309 (2001); Erratum 44, 2354 (2003).

${ }^{31}$ C. Tsallis, "Possible generalization of Boltzmann-Gibbs statistics," J. Stat. Phys. 52, 479 (1988).

${ }^{32}$ N. Leonenko, L. Pronzato, and V. Savani, "A class of Rényi information estimator for multi-dimensional densities," Ann. Stat. 40(4), 2153-2182 (2008).

${ }^{33}$ P. Jizba and T. Arimitsu, "The world according to Rényi: Thermodynamics of multifractal systems," Ann. Phys. 312, 17 (2004).

${ }^{34}$ J. S. Dehesa, S. López-Rosa, and D. Manzano, "Entropy and complexity analysis of $d$-dimension at quantum systems," in Statistical Complexities: Application to Electronic Structure, edited by K. D. Sen (Springer, Berlin, 2012), Chap. 5.

${ }^{35}$ I. Bialynicki-Birula and L. Rudnicki, "Entropic uncertainty relations in quantum physics," in Statistical Complexities: Application to Electronic Structure, edited by K. D. Sen (Springer, Berlin, 2011), Chap. 1.

${ }^{36}$ P. Jizba, J. A. Dunningham, and J. Joo, "Role of information theoretic uncertainty relations in quantum theory," Ann. Phys. 355, 87 (2015).

${ }^{37}$ M. J. W. Hall, “Universal geometric approach to uncertainty, entropy, and information,” Phys. Rev. A 59(4), 2602 (1999).

${ }^{38}$ I. Bialynicki-Birula, "Formulation of the uncertainty relations in terms of the Rényi entropies," Phys. Rev. A 74, 052101 (2006).

${ }^{39}$ S. Zozor and C. Vignat, "On classes of non-Gaussian asymptotic minimizers in entropic uncertainty principles," Physica A 375, 499 (2007).

${ }^{40}$ S. Zozor, M. Portesi, and C. Vignat, "Some extensions of the uncertainty principle," J. Phys. A 387, 4800 (2008).

${ }^{41}$ T. H. Koornwinder, R. Wong, R. Koekoek, and R. F. Swarttouw, "Orthogonal polynomials," in NIST Handbook of Mathematical Functions (Cambridge University Press, New York, 2010), Chap. 18.

${ }^{42}$ J. Avery, Hyperspherical Harmonics and Generalized Sturmmians (Kluwer Academic Publishers, New York, 2002).

${ }^{43}$ N. M. Temme, I. V. Toranzo, and J. S. Dehesa, "Entropic functionals of Laguerre and Gegenbauer polynomials with large parameters," J. Phys. A: Math. Theor. 50(21), 215206 (2017).

${ }^{44}$ N. M. Temme, "Uniform asymptotic methods for integrals," Indagationes Math. 24, 739-765 (2013).

${ }^{45}$ N. M. Temme, Asymptotic Methods for Integrals, Series in Analysis (World Scientific Publishing Co. Pte. Ltd., Hackensack, NJ, 2015). 